\title{
Tradução e adaptação do Check List RE-AIM para a realidade Brasileira
}

\section{Translation and adaptation of the RE-AIM Check List for Brazilian reality}

\section{AUTORES \\ Fabiana Almeida Brito ${ }^{1}$ (D) \\ Tânia Rosane Bertoldo Benedeti² (D) \\ Camila Tomicki ${ }^{2}$ (D) \\ Lisandra Maria Konrad ${ }^{2}$ iD \\ Paula Fabrício Sandreschi ${ }^{2}$ (D) \\ Sofia Wolker Manta ${ }^{2}$ (iD \\ Fabio Araujo Almeida ${ }^{1}$ (D) \\ 1 University of Nebraska Medical Center, College of Public Health, Department of Health Promotion, Social and Behavioral Health, Omaha, Nebraska, Estados Unidos. \\ 2 Universidade Federal de Santa Catarina, Programa de Pós-Graduação em Educação Física, Florianópolis, Santa Catarina, Brasil.}

\section{CONTATO}

\section{Fabiana Almeida Brito}

fabiana.silva@unmc.edu

Nebraska Medical Center, 984365, Omaha,

Nebraska, Estados Unidos. 68198-4365.

\section{DOI}

$10.12820 /$ rbafs.23e0033

\section{(1) $\Theta$}

Copyrioht: This is an open-access article distributed under the terms of the Creative Commons Attribution License ${ }^{\circledR}$, which permits unrestricted use, distribution, and reproduction in any medium, provided that the original author and source are credited.

\begin{abstract}
RESUMO
O objetivo foi traduzir e adaptar os itens de codificação relacionados às dimensões do modelo RE-AIM para auxiliar no planejamento e avaliação de programas de promoção da atividade física e saúde, bem como na análise da qualidade do relato em estudos de revisão sistemática. Foram realizadas equivalência conceitual, de itens e semântica por profissionais de saúde mono e bilíngues. Na análise conceitual e de itens foi realizada discussão entre especialistas e na semântica, tradução inversa (inglês para português) e reversa (português para inglês) dos itens. Posteriormente, foi avaliada a equivalência entre as retraduções e o conteúdo original. Dessa forma, foi apresentada a tradução e adaptação dos 54 itens que permite o planejamento e avaliação de programas e dos 21 itens para análise da qualidade das informações relatadas em evidências científicas. Espera-se contribuir para o planejamento e avaliação de programas ou revisões sistemáticas para promoção da atividade física e saúde.
\end{abstract}

Palavras-chave: Avaliação de programas; Revisão sistemática; RE-AIM; Atividade motora; Saúde. ABSTRACT

The goal was to translate and adapt the coding items related to the dimensions of the RE-AIM model to assist in the planning and evaluation of physical activity and health promotion programs as well as in the analysis of the quality of the report in systematic review studies. Mono and bilingual health professionals carried out conceptual, item and semantic equivalence. In the conceptual and item analysis specialists carried out a discussion and in the semantic one, an inverse (English to Portuguese) and a reverse (Portuguese to English) translation of the items were performed. Posteriorly, the equivalence between the retranslations and the original content was evaluated. Therefore, the translation and adaptation of the 54 items that allow the planning and evaluation of the programs were presented as well as the translation and adaptation of the 21 items necessary to the analysis of the quality of the information reported in scientific evidence. It is expected to contribute to programs planning and evaluation or their systematic review aiming at physical activity and health promotion.

Keywords: Program evaluation; Systematic review; RE-AIM; Physical activity; Health.

\section{Introdução}

A inatividade física é um grave problema de saúde pública ${ }^{1}$, que atinge um em cada três adultos mundialmente $^{2}$. Além da associação com o desenvolvimento de doenças crônicas não transmissíveis (DCNTs), ela é considerada o quarto fator de risco à mortalidade ${ }^{1}$ sendo responsável por 5,3 milhões de mortes em todo mundo ${ }^{2}$. Cerca de $13,7 \%$ da população adulta brasileira ( $\geq 18$ anos) é considerada fisicamente inativa ${ }^{3}$.

Como uma forma de mudar este cenário, em 2006 o Brasil propôs a Política Nacional de Promoção da
Saúde (PNPS) ${ }^{4}$ que contribuiu para promoção da atividade física. A partir disso, a atividade física foi inserida na agenda de promoção da saúde e passou a integrar o conjunto de iniciativas inseridas no Sistema Único de Saúde (SUS), em especial na Atenção Básica à Saúde (ABS) com a criação do Núcleo de Apoio à Saúde da Família (NASF) em 20085. No Brasil, cerca de 47\% dos programas que realizam promoção da atividade física são de caráter comunitário e no âmbito do SUS ${ }^{4}$. Entretanto as informações sobre o processo e o impacto destes programas são escassas ${ }^{4}$. 
De maneira geral, há informações sobre a efetividade dos programas para aumentar os níveis de atividade física. Contudo, a impossibilidade de tradução de tais propostas, ou a falta de descrições detalhadas dos procedimentos utilizados nos programas dificulta a compreensão dos métodos e também a possibilidade de replicação em outros contextos.

As revisões sistemáticas sobre programas de promoção de atividade física revelam falta de informação sobre os programas. Os estudos apontaram que a adoção foi relatada em $25 \%$ a $75 \%$ dos estudos, a implementação em $41 \%$ a $82 \%$, e a manutenção em apenas $10 \%$ e $11 \%$. Portanto, informações importantes são esquecidas ou pouco relatadas nos estudos, embora fundamentais para o planejamento, execução e êxito de tais estratégias ${ }^{6-9}$.

Para aumentar a probabilidade de sucesso em implementar programas e promover a disseminação em contexto de mundo real, é fundamental avaliar não somente a efetividade, mas os resultados relacionados a outras dimensões consideradas essenciais para a sustentabilidade desses programas ${ }^{9}$.

Com isso, o modelo RE-AIM foi desenvolvido para auxiliar na melhoria da qualidade dos relatos e fatores de validade interna e externa, afim de compreender o potencial impacto da saúde pública nos programas comportamentais ${ }^{10}$. Além da efetividade/ eficácia, o modelo especifica dimensões importantes como Adoção, Alcance, Implementação e Manutenção. Em 2013, foi traduzido e adaptado culturalmente à realidade brasileira visando contribuir, tanto em nível individual quanto organizacional, no planejamento, na avaliação e no monitoramento de intervenções ${ }^{11}$. Há, contudo, uma lacuna na forma de sistematização das informações coletadas por não haver um check list das dimensões que compõem o RE-AIM adaptado para a realidade brasileira.

Assim, a proposta deste artigo é traduzir e adaptar os itens de codificação relacionados às dimensões do modelo RE-AIM para auxiliar no planejamento e avaliação de programas de promoção da atividade física e saúde, bem como na análise da qualidade do relato em estudos de revisão sistemática.

\section{Método}

O RE-AIM auxilia na avaliação do impacto de programas de promoção à saúde pública por meio de cinco dimensões: Reach (Alcance), Effectiveness or Efficacy (Efetividade ou Eficácia), Adoption (Adoção), Implementation (Implementação) e Maintenance (Manutenção) ${ }^{10}$.
O instrumento de codificação do modelo RE-AIM contempla um check list de 54 itens (www.re-aim.org) para auxiliar no planejamento e avaliação de programas de promoção da atividade física e saúde. Para levantar dados de estudos por meio de revisões sistemáticas foi sugerido utilizar a versão reduzida de 21 itens validados $^{12}$ para cada uma das cinco dimensões do modelo: alcance $(n=5)$, efetividade/eficácia $(n=4)$, adoção $(n=$ $6)$, implementação $(n=3)$ e manutenção $(n=3)$.

A partir desses materiais existentes, adotou-se a metodologia para traduzir os itens de codificação de acordo com os protocolos utilizados no estudo de Almeida et al. ${ }^{11}$. As equivalências, conceitual e de itens, envolveram a discussão com especialistas (comitês) com o objetivo de explorar o instrumento de codificação original a fim de verificar sua relevância e aplicabilidade no contexto brasileiro. Além disso, um grupo de pesquisadores da área da saúde mono e bilíngues envolveram-se nos itens de codificação de cada dimensão considerando a estrutura do modelo RE-AIM traduzido.

A equivalência semântica incluiu as traduções inversa e reversa do instrumento de codificação de cada dimensão do modelo RE-AIM da sua língua original (inglês) para a língua portuguesa, por meio de dois tradutores brasileiros (nativos), especializados no ensino da língua inglesa e realizada a tradução reversa (retradução) do português para o inglês por dois brasileiros com naturalização americana que vivem nos Estados Unidos. Adicionalmente, foi avaliada a equivalência entre as retraduções e o instrumento original ${ }^{11}$.

Posteriormente, o primeiro grupo de especialistas (que participou da avaliação das equivalências conceitual e de itens) trabalhou criteriosamente na detecção e encaminhamento de discrepâncias semânticas das etapas anteriores e tal avaliação envolveu os tradutores e a equipe de pesquisa, responsáveis pela comparação das retraduções com o original.

Solucionados os problemas e realizadas modificações consensuais, foi criada a versão síntese do instrumento. Esta versão foi apresentada a outro grupo de pesquisadores, desta vez, multiprofissionais, para que observassem potenciais problemas de tradução e conceitos, além da clareza e adequação aos objetivos.

A partir disso, novas avaliações foram conduzidas pela equipe de pesquisa em conjunto com os especialistas multiprofissionais. Com base nas evidências encontradas nesse pré-teste, foi realizada a equivalência operacional referente aos aspectos de utilização do instrumento. Dessa forma, uma adaptação final do instru- 
mento foi realizada conforme apresentada nos resultados do presente estudo.

\section{Resultados}

Foi realizada a tradução e adaptação dos itens que compõem o check list. No Quadro 1 são apresentados os 54 itens e a versão reduzida com os 21 itens em destaque.
Com base nos 21 itens é possível verificar se os relatos apresentaram informações suficientes nas cinco dimensões do RE-AIM. Para análise, cada um dos itens deve ser pontuado de acordo com a presença (1 ponto) ou ausência (0 ponto) da informação, com o respectivo percentual, tendo como base o número total de estudos incluídos da revisão sistemática (Tabela 1).

Quadro 1 - Descrição e exemplos dos 54 itens do RE-AIM utilizados para auxiliar no planejamento e avaliação de programas de promoção da atividade física e saúde e 21 itens para avaliar a qualidade do relato de artigos de intervenção em revisões sistemáticas.

\begin{tabular}{|c|c|}
\hline Dimensão & Descrição e exemplo \\
\hline \multicolumn{2}{|r|}{ Alcance (Reach) } \\
\hline 1. Descrição da população-alvo** & $\begin{array}{l}\text { Descrição breve da população-alvo (não se restringir à amostra do estudo). } \\
\text { Ex.: A população incluiu todos os usuários da unidade básica de saúde (UBS) com mais de } 18 \text { anos de idade e que } \\
\text { não cumpriam recomendações de atividade física. }\end{array}$ \\
\hline $\begin{array}{l}\text { 2. Informações demográficas e } \\
\text { comportamentais da população-alvo }\end{array}$ & $\begin{array}{l}\text { Descrição das características mais específicas da população-alvo (não se restringir à amostra do estudo). } \\
\text { Ex.: } 70 \% \text { eram mulheres, } 52 \% \text { tem ensino fundamental completo, } 63 \% \text { eram aposentadas, } 80 \% \text { não cumpriam as } \\
\text { recomendações de prática de atividade física e 5\% eram fumantes. }\end{array}$ \\
\hline $\begin{array}{l}\text { 3. Método de identificação da população- } \\
\text { alvo** }\end{array}$ & $\begin{array}{l}\text { Descrição do processo pelo qual foi identificada a população para a participação no estudo. } \\
\text { Ex.: Todos os usuários da UBS foram identificados utilizando o prontuário eletrônico. }\end{array}$ \\
\hline 4. Critérios de inclusão** & $\begin{array}{l}\text { Descrição das características da população-alvo usadas para determinar se o participante potencial é elegível para } \\
\text { participar do estudo. } \\
\text { Ex.: Adultos sem diabetes e com sobrepeso (IMC } \geq 25 \mathrm{~kg} / \mathrm{m}^{2} \text { ) que apresentavam obesidade abdominal } \\
\text { (circunferência da cintura } \geq 102 \mathrm{~cm} \text { para homens e } \geq 88 \mathrm{~cm} \text { para mulheres). }\end{array}$ \\
\hline 5. Critérios de exclusão** & $\begin{array}{l}\text { Descrição das características que impediriam um participante potencial de ser elegível para participar. Também a } \\
\text { percentagem de excluídos pode ser reportada. } \\
\text { Ex.: Indivíduos que relataram um diagnóstico de diabetes ou que utilizavam medicação para baixar a glicose. }\end{array}$ \\
\hline 6. Estratégias de recrutamento & $\begin{array}{l}\text { Descrição dos métodos utilizados para recrutar os participantes para o estudo. } \\
\text { Ex.: Foram utilizados panfletos, apresentações em reuniões, meios de comunicação e divulgação "boca-a-boca" para } \\
\text { recrutar os participantes. }\end{array}$ \\
\hline $\begin{array}{l}\text { 7. Número de elegíveis e convidados } \\
\text { (expostos) }\end{array}$ & $\begin{array}{l}\text { Descrição do número total de pessoas que atenderam aos critérios de elegibilidade e foram contatadas para } \\
\text { participar do programa. } \\
\text { Ex.: Foram contatadas } 300 \text { pessoas para o estudo e } 250 \text { foram elegíveis. }\end{array}$ \\
\hline 8. Tamanho da amostra & $\begin{array}{l}\text { Descrição do número total de pessoas que concordaram em participar. } \\
\text { Ex.: } 200 \text { pessoas concordaram em participar do programa. }\end{array}$ \\
\hline 9. Taxa de participação** & $\begin{array}{l}\text { Proporção de participantes que aceitaram iniciar o programa. Calcula-se dividindo o tamanho da amostra pela } \\
\text { população-alvo e multiplica-se por } 100 \text {. } \\
\text { Ex.: } 200 \text { (número de pessoas que concordam em participar) / } 250 \text { (número de participantes que compunham a } \\
\text { população-alvo contatados para participação) x } 100=80 \% \text { de taxa de participação. }\end{array}$ \\
\hline 10. Custo do recrutamento & $\begin{array}{l}\text { Descrição do custo total e por estratégias de recrutamento. O valor pode refletir unidades monetárias e/ou de } \\
\text { tempo. Também pode ser relatado o custo por participante recrutado. } \\
\text { Ex.: O custo total para um programa que teve } 200 \text { participantes foi de } \mathrm{R} \$ 1.000,00 \text { com a estratégia de } \\
\text { recrutamento A (panfletos) e } \mathrm{R} \$ 200,00 \text { com a estratégia B (anúncios de jornal). Totalizando um custo de } \mathrm{R} \$ 6,00 \\
\text { por participante ( } \mathrm{R} \$ 1.200,00 / 200 \text { participantes). }\end{array}$ \\
\hline $\begin{array}{l}\text { 11. Utilização de métodos qualitativos } \\
\text { para aferir alcance }\end{array}$ & $\begin{array}{l}\text { Informações sobre aspectos não quantitativos do alcance. Observações em palavras, frases, descrições ou códigos. } \\
\text { Ex.: Alguns métodos comuns incluem entrevistas com informantes-chave (coordenador da UBS, por exemplo), } \\
\text { grupos de discussão, grupos focais ou até mesmo anotações de campo que fornecem informações sobre as } \\
\text { percepções, sentimentos, opiniões e experiências. }\end{array}$ \\
\hline \multicolumn{2}{|r|}{ Efetividade/Eficácia (Effectiveness/Efficacy) } \\
\hline 12. Resultados dos desfechos primários** & $\begin{array}{l}\text { Descrição dos resultados dos desfechos primários do estudo. } \\
\text { Ex.: Os participantes perderam, em média, } 1,5 \mathrm{~kg} \text { e aumentaram } 30 \text { minutos de atividade física após participação } \\
\text { no programa. }\end{array}$ \\
\hline 13. Relato de mediadores & $\begin{array}{l}\text { Descrição das variáveis que funcionam como mediadoras nas análises de efetividade e eficácia. Ou seja, } \\
\text { características modificáveis que podem ser manipuladas para uma maior efetividade. } \\
\text { Ex.: Ao analisar diferenças no apoio social e perda de peso entre os grupos intervenção e controle, as análises de } \\
\text { mediação buscam determinar se a mudança de suporte social foi o preditor proximal de perda de peso. Então o } \\
\text { teste de mediação de Baron \& Kenny indicou que os efeitos do programa (perda de peso) foram mediados pelo } \\
\text { apoio social. }\end{array}$ \\
\hline
\end{tabular}

Continua... 
... continua

\begin{tabular}{|c|c|}
\hline Dimensão & Descrição e exemplo \\
\hline 14. Relato de moderadores & $\begin{array}{l}\text { Descrição das variáveis que funcionam como moderadoras nas análises de efetividade e eficácia. Ou seja, } \\
\text { características não modificáveis (como sexo, raça ou idade) que podem influenciar na efetividade. } \\
\text { Ex.: Os participantes afro-americanos na condição de intervenção perderam } 10 \% \text { do seu peso corporal em } \\
\text { comparação com os participantes brancos que perderam uma média de } 5 \% \text { do peso corporal. }\end{array}$ \\
\hline $\begin{array}{l}\text { 15. Intenção de tratamento ou presentes } \\
\text { no acompanhamento** }\end{array}$ & $\begin{array}{l}\text { Análise de intenção de tratar: quando os participantes do estudo foram analisados nos grupos aos quais foram } \\
\text { randomizados, independentemente de receberem ou aderirem ao programa. Descrição de uma imputação que foi } \\
\text { utilizada para contabilizar dados perdidos na análise. } \\
\text { Ex.: O valor de baseline foi imputado para todos os participantes que não compareceram à consulta de } \\
\text { acompanhamento para que todos os participantes pudessem ser incluídos na análise. } \\
\text { Análise dos presentes no acompanhamento: descrição da análise quando apenas os participantes que completaram } \\
\text { a avaliação de acompanhamento foram incluídos. } \\
\text { Ex.: Somente os participantes que completaram as medidas no início do estudo e o acompanhamento foram } \\
\text { incluídos na análise. }\end{array}$ \\
\hline 16. Medidas de qualidade de vida** & $\begin{array}{l}\text { Descrição de uma medida de qualidade de vida. Pode-se considerar quando os artigos se referirem a variáveis de } \\
\text { bem-estar ou satisfação com a vida. } \\
\text { Ex.: SF-12v2, uma versão curta do questionário de qualidade de vida SF-36; Impact of Weight on Quality of Life- } \\
\text { Lite (IWQOL-L), Patient Health Questionnaire-9 (PHQ-9). Também podem ser utilizadas perguntas, como } \\
\text { "tomando como referências as duas últimas semanas, como você avalia sua qualidade de vida?". }\end{array}$ \\
\hline $\begin{array}{l}\text { 17. Consequências não intencionais } \\
\text { (negativas) nos resultados }\end{array}$ & $\begin{array}{l}\text { Resultados ou produtos do programa que não foram previstos e podem ter causado danos não intencionais. } \\
\text { Ex.: Em um programa de promoção de atividade física, os participantes do sexo feminino tiveram um aumento da } \\
\text { taxa de lesões. }\end{array}$ \\
\hline $\begin{array}{l}\text { 18. Taxa de abandono (na conclusão do } \\
\text { programa)** }\end{array}$ & $\begin{array}{l}\text { Proporção de participantes que desistiram do programa. Calcula-se dividindo o número de participantes que não } \\
\text { completaram o programa pelo número de participantes que iniciaram o programa e multiplica-se por } 100 . \\
\text { Ex.: } 20 \text { (não completaram o programa) / } 100 \text { (iniciaram o programa) x 100 = 20\% de abandonos/perdas. }\end{array}$ \\
\hline 19. Custo-efetividade & $\begin{array}{l}\text { Informações entre os valores para o custo do programa e o que de positivo pode resultar dele. Ou seja, o custo total } \\
\text { do programa dividido por cada sucesso (por exemplo, por cada quilo perdido ou por cada minuto de atividade fisica } \\
\text { aumentado). } \\
\text { Ex.: A nova estratégia pode economizar R } \$ 1.000,00 \text { por participante por ano quando comparado com a prática } \\
\text { atual. }\end{array}$ \\
\hline $\begin{array}{l}\text { 20. Medidas qualitativas da efetividade/ } \\
\text { eficácia }\end{array}$ & $\begin{array}{l}\text { Informações sobre obtenção de feedback dos participantes sobre o grau em que eles sentiram que o programa foi } \\
\text { efetivo/eficaz. } \\
\text { Ex.: Alguns métodos comuns incluem grupos focais, entrevistas, diários (texto/fotos). }\end{array}$ \\
\hline \multicolumn{2}{|r|}{ Adoção (Adoption) } \\
\hline $\begin{array}{l}\text { 21. Critérios de inclusão/exclusão dos } \\
\text { locais }\end{array}$ & $\begin{array}{l}\text { Descrição das características adotadas para determinar se um local potencial é elegível para participação ou aquelas } \\
\text { que impediriam um potencial de ser elegível para participar. } \\
\text { Ex.: As UBS precisavam ter pelo menos mil usuários cadastrados. Porém, serão excluídas aquelas localizadas na } \\
\text { zona rural. }\end{array}$ \\
\hline $\begin{array}{l}\text { 22. Número de locais elegíveis e } \\
\text { convidados (expostos) }\end{array}$ & $\begin{array}{l}\text { Descrição do número total de locais que atenderam aos critérios de elegibilidade e foram contatadas para participar } \\
\text { do programa. } \\
\text { Ex.: Foram contatadas } 50 \text { locais para o estudo e } 42 \text { foram elegíveis. }\end{array}$ \\
\hline 23. Número de locais participantes & $\begin{array}{l}\text { Descrição do número total de locais que concordaram em participar. } \\
\text { Ex.: } 20 \text { locais concordaram em participar do programa. }\end{array}$ \\
\hline 24. Taxa de participação dos locais** & $\begin{array}{l}\text { Proporção de locais que participaram. Calcula-se dividindo o número de locais que participaram pelo número de } \\
\text { elegíveis, que atendiam aos critérios de inclusão e exclusão, e multiplica-se por } 100 \text {. } \\
\text { Ex.: } 20 \text { (número de locais que concordaram em participar) / } 44 \text { (número de locais elegíveis) x } 100=45 \% \text { de taxa de } \\
\text { participação. }\end{array}$ \\
\hline 25. Identificação do local & $\begin{array}{l}\text { Identificação do local de implantação do programa. } \\
\text { Ex.: O programa foi adotado pelas UBS de um determinado município. }\end{array}$ \\
\hline 26. Método de identificação do local & $\begin{array}{l}\text { Descrição do processo pelo qual o local foi identificado para a participar do estudo. } \\
\text { Ex.: As UBS foram identificadas de acordo com informações repassadas pela Secretaria Municipal de Saúde. }\end{array}$ \\
\hline $\begin{array}{l}\text { 27. Descrição do local que foi } \\
\text { desenvolvido o program*** }\end{array}$ & $\begin{array}{l}\text { Descrição das características do local do programa, como recursos disponíveis, informação sobre o pessoal, número } \\
\text { de locais elegíveis, ambiente de trabalho. } \\
\text { Ex.: Ocorreu em uma UBS de aproximadamente } 400 \mathrm{~m}^{2} \text { que tinha uma recepção, uma sala de procedimentos, uma } \\
\text { sala de vacinação, uma copa, três consultórios, uma recepção com sala de espera, dois banheiros, um almoxarifado, } \\
\text { uma sala de observação e uma sala de reuniões. }\end{array}$ \\
\hline $\begin{array}{l}\text { 28. Critério de inclusão/exclusão dos } \\
\text { membros da equipe** }\end{array}$ & $\begin{array}{l}\text { Descrição das características que foram consideradas para determinar o potencial do membro da equipe para } \\
\text { participação no programa. } \\
\text { Ex.: Possuir graduação em educação física. }\end{array}$ \\
\hline
\end{tabular}

Continua... 
... continua

\begin{tabular}{|c|c|}
\hline Dimensão & Descrição e exemplo \\
\hline $\begin{array}{l}\text { 29. Membros da equipe elegíveis e } \\
\text { convidados }\end{array}$ & $\begin{array}{l}\text { Descrição do número de membros da equipe de saúde que preencheram os critérios de elegibilidade e foram } \\
\text { convidados para auxiliar no programa. } \\
\text { Ex.: } 100 \text { profissionais preencheram os critérios de elegibilidade e todos foram convidados a participar. }\end{array}$ \\
\hline $\begin{array}{l}\text { 30. Membros da equipe que aceitaram } \\
\text { participar }\end{array}$ & $\begin{array}{l}\text { Total de membros da equipe que aceitaram participar. } \\
\text { Ex.: } 20 \text { profissionais aceitaram participar. }\end{array}$ \\
\hline $\begin{array}{l}\text { 31. Taxa de participação dos membros } \\
\text { da equipe } e^{* *}\end{array}$ & $\begin{array}{l}\text { Proporção de membros da equipe que participaram. Calcula-se dividindo o número de profissionais que } \\
\text { participaram pelo número de elegiveis, que atendiam aos critérios de inclusão e exclusão, e multiplica-se por } 100 . \\
\text { Ex.: } 20 \text { (número de profissionais que concordou em participar) / } 100 \text { (número de profissionais elegíveis) x 100 = } \\
20 \% \text { de taxa de participação. }\end{array}$ \\
\hline $\begin{array}{l}\text { 32. Método de identificação dos } \\
\text { membros da equipe** }\end{array}$ & $\begin{array}{l}\text { Descrição do processo de identificação dos membros da equipe elegiveis. } \\
\text { Ex.: Todos os membros da equipe do local do programa foram identificados pelos supervisores no local com base } \\
\text { nos critérios de elegibilidade. }\end{array}$ \\
\hline $\begin{array}{l}\text { 33. Média de membros participantes } \\
\text { por local }\end{array}$ & $\begin{array}{l}\text { Cálculo do número médio de participantes nos locais do programa. } \\
\text { Ex.: } 20 \text { (número de profissionais que participaram) / } 20 \text { (número de locais que participaram = } 1 \text {. } \\
\text { Participou, em média, um profissional por UBS. }\end{array}$ \\
\hline $\begin{array}{l}\text { 34. Nível de conhecimento dos membros } \\
\text { da equipe** }\end{array}$ & $\begin{array}{l}\text { Descrição de treinamento ou formação educacional dos membros da equipe para atuar no programa. } \\
\text { Ex.: Grau e/ou titulação dos membros da equipe. Como graduação em educação física, mestrado, doutorado, etc. }\end{array}$ \\
\hline 35. Mensuração de custo para adoção & $\begin{array}{l}\text { Descrição do custo para estratégias de adoção em todos os níveis do programa (como hora dos profissionais, } \\
\text { divulgação, etc). } \\
\text { Ex.: O custo com panfletos de divulgação foi de } \mathrm{R} \$ 1.000,00 \text {. }\end{array}$ \\
\hline \multicolumn{2}{|r|}{ Implementação (Implementation) } \\
\hline 36. Teorias & $\begin{array}{l}\text { Descrição de teorias ou princípios utilizados para desenvolver o programa. } \\
\text { Ex.: Esse programa foi desenvolvido baseada na Teoria Social Cognitiva de Albert Bandura. }\end{array}$ \\
\hline 37. Número de contatos*** & $\begin{array}{l}\text { Descrição do total de encontros com os participantes do programa. Incluir encontros presenciais, telefonemas, } \\
\text { boletins informativos, etc. } \\
\text { Ex.: O programa consistiu em } 12 \text { encontros presenciais, além de } 12 \text { contatos telefônicos. }\end{array}$ \\
\hline 38. Frequência dos contatos* ${ }^{* *}$ & $\begin{array}{l}\text { Descrição da frequencia que os contatos para o programa ocorreram ao longo do programa. } \\
\text { Ex.: Os encontros presenciais ocorriam semanalmente, todas as quintas-feiras. Já, os telefonemas eram realizados } \\
\text { semanalmente, porém às segundas-feiras. O programa teve duração total de } 3 \text { meses. }\end{array}$ \\
\hline 39. Duração dos contatos*** & $\begin{array}{l}\text { Descrição da duração de cada contato do programa. } \\
\text { Ex.: Os encontros presenciais tinha duração de } 50 \text { minutos. As primeiras } 4 \text { chamadas telefônicas duraram cerca de } \\
15 \text { minutos cada, as demais duraram cerca de } 10 \text { minutos cada. }\end{array}$ \\
\hline $\begin{array}{l}\text { 40. Medida em que o protocolo foi } \\
\text { executado conforme o esperado** }\end{array}$ & $\begin{array}{l}\text { Descrição da fidelidade ao protocolo do programa. } \\
\text { Ex.: Com base em uma lista dos componentes avaliados pelos membros da equipe que realizaram o programa, } 80 \% \\
\text { do conteúdo foi aplicado tal qual se pretendia. }\end{array}$ \\
\hline $\begin{array}{l}\text { 41. Cumprimento do planejamento } \\
\text { entre locais e equipe que realizaram o } \\
\text { programa }\end{array}$ & $\begin{array}{l}\text { Descrição do grau de similaridades entre os locais e membros da equipe do programa. } \\
\text { Ex.: } 5 \text { profissionais de educação física realizaram o programa, três deles cumpriram } 80 \% \text { do planejamento do } \\
\text { programa, os outros dois } 100 \% \text {. }\end{array}$ \\
\hline 42. Comparecimento dos participantes & $\begin{array}{l}\text { Informações de comparecimento dos participantes que concluíram o programa, em média. } \\
\text { Ex.: Os participantes compareceram, em média, a } 10 \text { dos } 12 \text { encontros do programa. }\end{array}$ \\
\hline 43. Medidas de custo** & $\begin{array}{l}\text { Descrição de custos associados com a implementação inicial do programa e sua subsequente continuação. } \\
\text { Ex.: Foram gastos } R \$ 1.000,00 \text { com a compra inicial de materiais, } R \$ 2.000,00 \text { com a compra de computadores, e } \\
R \$ 3.500,00 \text { com a contratação de novos profissionais. Durante o programa foram gastos } R \$ 200,00 \text { por mês com } \\
\text { aluguel do espaço físico e } R \$ 50,00 \text { com transporte. }\end{array}$ \\
\hline 44. Métodos qualitativos de avaliação & $\begin{array}{l}\text { Descrição da utilização de métodos qualitativos para compreender o processo de implementação. } \\
\text { Ex.: Grupos focais e entrevistas. }\end{array}$ \\
\hline \multicolumn{2}{|r|}{ Manutenção (Maintenance) } \\
\hline 45. Continuidade do programa** & $\begin{array}{l}\text { Descrição da continuação do programa após a conclusão da pesquisa. } \\
\text { Ex.: A Secretaria Municipal de Saúde continua oferecendo o programa um ano após o término do presente estudo. }\end{array}$ \\
\hline 46. Razões para a descontinuidade & $\begin{array}{l}\text { Razões para a descontinuidade do programa (se não houver continuidade). } \\
\text { Ex.: O programa não foi mais oferecido para os usuários pois a Secretaria Municipal de Saúde decidiu suspendê-lo } \\
\text { por não ter condições financeiras de mantê-lo. }\end{array}$ \\
\hline 47. Modificações no programa & $\begin{array}{l}\text { Descrição de quaisquer alterações que foram realizadas no protocolo original do programa. } \\
\text { Ex.: O programa começou a ser oferecido quinzenalmente ao invés de semanalmente. }\end{array}$ \\
\hline 48. Institucionalização do programa & $\begin{array}{l}\text { Descrição da forma como o programa foi integrado ao sistema por meio de métodos como mudanças políticas, } \\
\text { mudanças na descrição dos cargos e funções. } \\
\text { Ex.: A prefeitura municipal contratou um profissional para que atuasse exclusivamente na gestão do programa nas UBS. }\end{array}$ \\
\hline
\end{tabular}

Continua... 
... continua

\begin{tabular}{|ll|}
\hline Dimensão & Descrição e exemplo \\
\hline 49. Taxa de abandono organizacional & $\begin{array}{l}\text { Proporção de locais que desistiram de oferecer o programa. Calcula-se dividindo o número de locais que pararam } \\
\text { de oferecer o programa pelo número de locais que iniciaram o programa e multiplica-se por } 100 . \\
\text { Ex.: 2 (desistiram de oferecer o programa) / } 10 \text { (iniciaram o programa) x } 100=20 \% \text { de abandonos. }\end{array}$ \\
\hline $\begin{array}{l}\text { 50. Alinhamento com a missão/ } \\
\text { princípios/diretrizes da organização }\end{array}$ & $\begin{array}{l}\text { Declaração explicita de como o modelo do programa se alinha com a missão/princípios/diretrizes da organização } \\
\text { onde foram aplicados seus valores. } \\
\text { Ex.: O programa foi projetado para se alinhar com a missão organizacional do local (cada local tem a sua missão). }\end{array}$ \\
\hline $\begin{array}{l}\text { 51. Avaliação de resultados após a } \\
\text { conclusão do programa (duração do } \\
\text { acompanhamento)** }\end{array}$ & $\begin{array}{l}\text { Descrição das medidas (avaliações) de acompanhamento dos resultados disponíveis dos indivíduos algum tempo } \\
\text { após o término do programa. } \\
\text { Ex.: 6 meses após o término do programa os participantes haviam retornado aos níveis iniciais de atividade física. }\end{array}$ \\
\hline $\begin{array}{l}\text { 52. Taxa de abandono individual (durante } \\
\text { o acompanhamento*** }\end{array}$ & $\begin{array}{l}\text { Proporção de participantes que desistiram do programa. Calcula-se dividindo o número de participantes que foram } \\
\text { perdidos no acompanhamento pelo número de participantes que iniciaram o programa e multiplica-se por 100. } \\
\text { Ex.: 20 (foram perdidos no acompanhamento) / 100 (iniciaram o programa) x 100 = 20\% de abandonos. }\end{array}$ \\
\hline 53. Métodos qualitativos de avaliação & $\begin{array}{l}\text { Descrição da utilização de métodos qualitativos utilizados para compreender o processo de manutenção de } \\
\text { mudanças no resultado principal em nível individual. } \\
\text { Ex.: Grupos focais, entrevistas. }\end{array}$ \\
\hline 54. Custos da manutenção & $\begin{array}{l}\text { Descrição dos custos para manter o programa. } \\
\text { Ex.: Foram gastos R\$ 2.000,00 com a contratação de profissionais para realização das avaliações de manutenção. }\end{array}$ \\
\hline
\end{tabular}

* Os itens 37, 38 e 39 são avaliados de forma conjunta quando se trata do check list de 21 itens, devendo ser analisado como um único item (item 16 da Tabela 1). * Itens para avaliar a qualidade do relato de artigos de intervenção em revisões sistemáticas.

Tabela 1 - Modelo de tabela para descrever a proporção das dimensões do RE-AIM em estudos de revisão sistemática (21 itens).

Dimensão

Alcance $($ Reach)

1. Descrição da população-alvo

2. Método de identificação da população-alvo

3. Critérios de inclusão

4. Critérios de exclusão

5. Taxa de participação

\section{Efetividade/Eficácia (Effectiveness/Efficacy)}

6. Resultados dos desfechos primários

7. Intenção de tratamento ou presentes no acompanhamento

8. Medidas de qualidade de vida

9. Taxa de abandono (na conclusão do programa)

\section{Adoção(Adoption)}

10. Taxa de participação dos locais

11. Descrição do local que foi desenvolvido o programa

12. Critério de inclusão/exclusão dos membros da equipe

13. Taxa de participação dos membros da equipe

14. Método de identificação dos membros da equipe

15. Nível de conhecimento dos membros da equipe

Implementação (Implementation)

16. Número/frequência/duração dos contatos

17. Medida em que o protocolo foi executado conforme o esperado

18. Medidas de custo

\section{Manutenção (Maintenance)}

19. Continuidade do programa

20. Avaliação de resultados após a conclusão do programa (duração do acompanhamento)

21. Taxa de abandono individual (durante o acompanhamento)

* Quantidade de estudos que reportam determinado item. n 
Tabela 2 - Modelo de tabela para organização e apresentação dos resultados de estudos incluídos em revisões sistemáticas para análise dos 21 itens do RE-AIM.

\begin{tabular}{|c|c|c|c|c|c|c|c|}
\hline Estudos & $\begin{array}{c}\mathrm{R} \\
\operatorname{máx}=5^{*}\end{array}$ & $\begin{array}{c}\mathrm{E} \\
\operatorname{máx}=4^{*}\end{array}$ & $\begin{array}{c}\mathrm{A} \\
\operatorname{máx}=6^{*}\end{array}$ & $\begin{array}{c}\mathrm{I} \\
\operatorname{máx}=3^{*}\end{array}$ & $\begin{array}{c}\mathrm{M} \\
\operatorname{máx}=3^{*}\end{array}$ & $\begin{array}{c}\text { Total } \\
\text { máx = } 21\end{array}$ & Qualidade ${ }^{* *}$ \\
\hline Estudo 1 & [pontuação] & [pontuação] & [pontuação] & [pontuação] & [pontuação] & [somatório] & [Baixa/Moderada/Alta] \\
\hline Estudo 2 & [pontuação] & [pontuação] & [pontuação] & [pontuação] & [pontuação] & [somatório] & [Baixa/Moderada/Alta] \\
\hline Estudo 3 & [pontuação] & [pontuação] & [pontuação] & [pontuação] & [pontuação] & [somatório] & [Baixa/Moderada/Alta] \\
\hline Estudo 4 & [pontuação] & [pontuação] & [pontuação] & [pontuação] & [pontuação] & [somatório] & [Baixa/Moderada/Alta] \\
\hline Estudo 5 & [pontuação] & [pontuação] & [pontuação] & [pontuação] & [pontuação] & [somatório] & [Baixa/Moderada/Alta] \\
\hline
\end{tabular}

$\mathrm{R}=$ Alcance $($ Reach); E = Efetividade (Effectiveness/Efficacy); A = Adoção (Adoption); I = Implementação (Implementation); M = Manutenção (Maintenance). * Representa o número de itens relatados nos estudos incluídos por dimensão; ** Qualidade baixa = 0 a 7; Qualidade moderada $=8$ a 14; Qualidade alta $=15$ a 21;** Fonte: Traduzido e adaptado de Akers, Estabrooks \& Davy ${ }^{12}$.

Por exemplo, com relação ao item 1 do alcance, se foram incluídos 10 estudos na revisão sistemática, e desses, 3 relatarem a descrição da população-alvo, 30,0\% dos estudos atenderam a esse item.

Posteriormente, para cada estudo incluído na revisão sistemática, é realizado um somatório das pontuações obtidas com base nos 21 itens. $\mathrm{O}$ escore gerado permite identificar um somatório total e por dimensão do RE-AIM que foram analisadas. Após o cálculo do escore, sugere-se a seguinte análise para a classificação de qualidade das informações relatadas: a) estudos de intervenção com baixa qualidade ( 0 - 7 pontos), b) moderada qualidade (8 - 14 pontos) e c) alta qualidade (15 - 21 pontos) - Tabela 2 .

\section{Discussão}

Foram traduzidos e adaptados os itens de codificação relacionados às dimensões do modelo RE-AIM para auxiliar no planejamento e avaliação de programas de promoção da atividade física e saúde por meio de 54 itens, bem como para a análise da qualidade do relato em estudos de revisão sistemática por meio de 21 itens.

A análise dos 54 itens das dimensões do RE-AIM (Alcance, Efetividade/Eficácia, Adoção, Implementação e Manutenção) permite aos pesquisadores além de orientações para o planejamento de programas, possibilita a avaliação e replicação em diferentes contextos. $\mathrm{Na}$ sua forma reduzida, os 21 itens propõem a análise da qualidade das informações relatadas em estudos com proposta de revisão sistemática. Com isso, é possível identificar quais as dimensões são frequentemente relatadas, além de atribuir uma pontuação para qualidade dos estudos com intervenções em atividade física e saúde.

A avaliação de programas em promoção da atividade física e saúde torna-se fundamental para a análise da replicabilidade e qualidade das propostas e evidências científicas. A falta de metodologias e análises contextuais complexas tem evidenciado a baixa transição da evidência para a prática e, consequentemente, a dificuldade de transformar resultados de pesquisas com alto impacto clínico em políticas para saúde pública ${ }^{6,7,10}$. Nesse sentido, a avaliação interna e externa das evidências, com base no modelo RE-AIM poderá clarificar e promover o melhoramento do planejamento e qualidade dos relatos para intervenções em promoção de saúde em nosso país ${ }^{10,11}$.

Para análise de intervenções e seus respectivos impactos na saúde faz-se necessário uma avaliação multidimensional e criteriosa ${ }^{10}$. Estudos que utilizaram o modelo RE-AIM como forma de avaliar intervenções verificaram que poucos estudos contemplaram a análise de todas as dimensões e, quando presente, foram relatadas de forma incompleta ${ }^{6-9,13,14}$.

Quanto a isso, observa-se que contemplar o RE-AIM em sua forma completa nas intervenções, é dificultada pela necessidade de planejamento, coleta de dados com uso de diferentes instrumentos para cada uma das dimensões. Além, das necessidades de recursos (humanos, físicos, instrumentais, etc.) da comunidade, das organizações, do ambiente - comunitário, clínico ou corporativo - bem como das características da intervenção, populações atingidas e resultados desejados ${ }^{14}$. Consequentemente, isto acarreta altos custos financeiros à pesquisa atingindo principalmente países de baixa e média renda como o Brasil ${ }^{14}$. De qualquer forma, quando as dimensões do RE-AIM não são analisadas de forma conjunta, não há como prever em quais destas, as estratégias devem se concentrar para melhor qualidade das intervenções, impedindo também a comparabilidade entre estudos ${ }^{10,14}$. Recomenda-se aos pesquisadores clareza ao disseminar informações de suas investigações quando tratam de uma dimensão de forma isolada, referindo, especialmente, os motivos pela exclusão das demais ${ }^{13}$.

Em estudos de revisão sistemática, sugere-se aos 
pesquisadores pontuar precisamente de que forma as dimensões do RE-AIM têm sido atendidas nos relatos, para que assim, com base em evidências, seja possível discutir em futuras pesquisas ${ }^{8}$. Estes estudos são pertinentes por evidenciar, sistematicamente, o impacto das intervenções em promoção da atividade física e saúde nos diferentes contextos sociais, culturais e econômi$\cos ^{4,6,7}$. A replicabilidade de intervenções efetivas e com boa qualidade no cenário mundial, poderá possivelmente contribuir para a reversão do cenário da inatividade física em direção à comportamentos ativos e saudáveis.

Sendo assim, a avaliação de programas em promoção da atividade física e saúde, com base em check lists do modelo RE-AIM permite analisar desde o planejamento e avaliação de programas (54 itens), a qualidade das informações em revisões sistemáticas (21 itens). Ressalta-se que pequenas adaptações na extração e análise de dados podem ser realizadas para adequação ao objetivo do estudo, porém não deve ser alterada a natureza das dimensões. Espera-se com isso, contribuir para futuros planejamentos e avaliações de programas em promoção da atividade física e saúde, afim de aprimorar a qualidade dos seus relatos para que sejam possíveis de serem replicáveis em diferentes contextos.

\section{Conflito de interesses}

Os autores declaram não haver conflito de interesse.

\section{Contribuição dos autores}

Brito FA, participou da concepção inicial do estudo, redação e revisão crítica do texto. Tomicki C, Konrad LM, Sandreschi PF e Manta SW, foram responsáveis pela busca da literatura e redação do texto. Almeida FA e Benedetti TRB, realizaram a revisão crítica dos conteúdos e aprovação da versão final a ser publicada.

\section{Referências}

1. Kohl HW, Craig CL, Lambert EV, Inoue S, Alkandari JR, Leetongin $\mathrm{G}$, et al. The pandemic of physical inactivity: global action for public health. Lancet. 2012;380(9838):294-05.

2. Hallal PC, Andersen LB, Bull FC, Guthold R, Haskell W, Ekelund U. Global physical activity levels: surveillance progress, pitfalls, and prospects. Lancet. 2012;380(9838):247-57.
3. Ministério da Saúde (Brasil). Secretaria de Vigilância em Saúde. Departamento de Vigilância de Doenças e Agravos não Transmissíveis e Promoção da Saúde. VIGITEL Brasil 2016: vigilância de fatores de risco e proteção para doenças crônicas por inquérito telefônico: estimativas sobre frequência e distribuição sociodemográfica de fatores de risco e proteção para doenças crônicas nas capitais dos 26 estados brasileiros e no Distrito Federal em 2016. Brasília: Ministério da Saúde, 2017.

4. Becker LA, Gonçalves PB, Reis RS. Programas de promoção da atividade física no Sistema Único de Saúde brasileiro: revisão sistemática. Rev Bras Ativ Fís Saúde. 2016;21(2):110-22.

5. Ministério da Saúde (Brasil). Portaria no 154, de 24 de janeiro de 2008. Cria os Núcleos de Apoio à Saúde da Família NASF. Diário Oficial da União 04 mar 2008; Seção1.

6. Galaviz KI, Harden SM, Smith E, Blackman KCA, Berrey LM, Mama SK, et al. Physical activity promotion in Latin American populations: a systematic review on issues of internal and external validity. Int J Behav Nutr Phys Act. 2014;11(77):1-13.

7. Harden SM, Gaglio B, Shoup JA, Kinney KA, Johson SB, Brito F, et al. Fidelity to and comparative results across behavioral interventions evaluated through the RE-AIM framework: a systematic review. Syst Rev. 2015;4:155.

8. Konrad LM, Tomicki C, Silva MC, Benedetti TRB, Almeida FA. Avaliação de programas de mudança de comportamento usando a ferramenta RE-AIM: um estudo de revisão sistemática. Rev Bras Ativ Fís Saúde. 2017:22(5):448-58.

9. Forman J, Heisler M, Damschroder LJ, Kaselitz E, Kerr EA. Development and application of the RE-AIM Quest mixed methods framework for program evaluation. Prev Med Rep. 2017;(4)6:322-28.

10. Glasgow RE, Vogt TM, Boles SM. Evaluating the public health impact of health promotion interventions: the REAIM framework. Am J Public Health. 1999;89(9):1322-27.

11. Almeida FA, Brito. FA, Estabrooks PA. Modelo RE-AIM: Tradução e adaptação cultural para o Brasil. Rev. Fam Ciclos Vida Saúde Contexto Soc. 2013;1(1):6-16.

12. Akers J, Estabrooks, PA, Davy BM. Translational research: bridging the gap between long-term weight loss maintenance research and practice.J Am Diet Assoc. 2010;110(10):1511-22.

13. Lee RE, Galavíz KI, Soltero EG, Rosales Chavez J, Jauregui E, Lévesque L, et al. Applying the RE-AIM conceptual framework for the promotion of physical activity in low- and middle-income countries. Rev. Latino-Am Enfermagem. 2017;25:e2923.

14. Harden SM, Smith ML, Ory MG, Smith-Ray L, Estabrooks PA, Glasglow RE. RE-AIM in Clinical, Community, and Corporate Settings: Perspectives, Strategies, and Recommendations to Enhance Public Health Impact Front Public Health. 2018:6:71. realidade Brasileira. Rev Bras Ati Fis Saúde. 2018;23:e0033. DOI: 10.12820/rbafs.23e0033 